\title{
Approximated Generalized Torques by the Hydrodynamic Forces Acting on Legs of an Underwater Walking $\operatorname{Robot}^{\dagger}$
}

\author{
Bong-Huan Jun*, Hyungwon Shim, and Pan-Mook Lee \\ Ocean Engineering Department, Korea Ocean Research and Development Institute, Daejeon 305-343, Korea
}

(Manuscript Received September 24, 2011; Revised October 15, 2011; Accepted November 9, 2011)

\begin{abstract}
In this paper, we present the concept and main mission of the Crabster, an underwater walking robot. The main focus is on the modeling of drag and lift forces on the legs of the robot, which comprise the main difference in dynamic characteristics between on-land and underwater robots. Drag and lift forces acting on the underwater link are described as a function of the relative velocity of the link with respect to the fluid using the strip theory. Using the translational velocity of the link as the rotational velocity of the joint, we describe the drag force as a function of joint variables. Generalized drag torque is successfully derived from the drag force as a function of generalized variables and its first derivative, even though the arm has a roll joint and twist angles between the joints. To verify the proposed model, we conducted drag torque simulations using a simple Selective Compliant Articulated Robot Arm.
\end{abstract}

Keywords: Crabster robot, Generalized drag torque, Hydrodynamic forces, Multi-legged robot, Underwater walking robot

\section{Introduction}

Most underwater vehicles use screws for propulsion due to the simple and well-studied mechanism. However, we noted 2 drawbacks of screw-type vehicles, the purpose of which is hovering and performing underwater manipulation. The first drawback is motion instability in the high tide area, which prevents precise localization, accurate manipulation, and clear acoustic imaging. The other drawback we noted is that the flow from the screw disturbs seabed sediment in the deep-sea environments in which scientists attempt to perform in situ inspections. To remedy these 2 drawbacks, here we present a new concept of an underwater robot named Crabster, which flies across the seabed like a crab and walks in high tide areas like a lobster. "Crabster" is compound word composed of "crab" and "lobster." A new project funded by the

\footnotetext{
tThis paper was presented at the OCEANS 2011 conference, Santander, Spain, in June 2011.

*Corresponding author. Tel.: +82-42-866-3812, Fax.: +82-42-3819.

E-mail address: bhjeon@moeri.re.kr.

Copyright $@$ KSOE 2011.
}

Ministry of Land \& Transportation and Maritime Affairs was launched in Korea in July 2010 to develop the Crabster robot.

Since the density of water is $1,000 \times$ higher than that of air, the hydrostatic and dynamic forces on a robot moving in water cannot be ignored. The consideration of hydrodynamic forces is one of the main elements in differentiating and characterizing the underwater robot from on-land robots. Several methods are used to analyze hydrodynamic forces such as those approaches using experiments, numerical analysis tools, and empirical formulas. L'evesque and Richard [1] presented an approximation method to compute the drag torque of a submerged square beam using Hoerner's crossflow principle for the pressure drag forces acting on the beam translating at an angle to the free stream. They then estimated the drag force on a segment of square beam and approximated the drag force of a long beam using the strip theory. Yuh [2] and Fossen [3] identified significant forces on a rigid body underwater, and Ioi and Itoh [4] extended the 


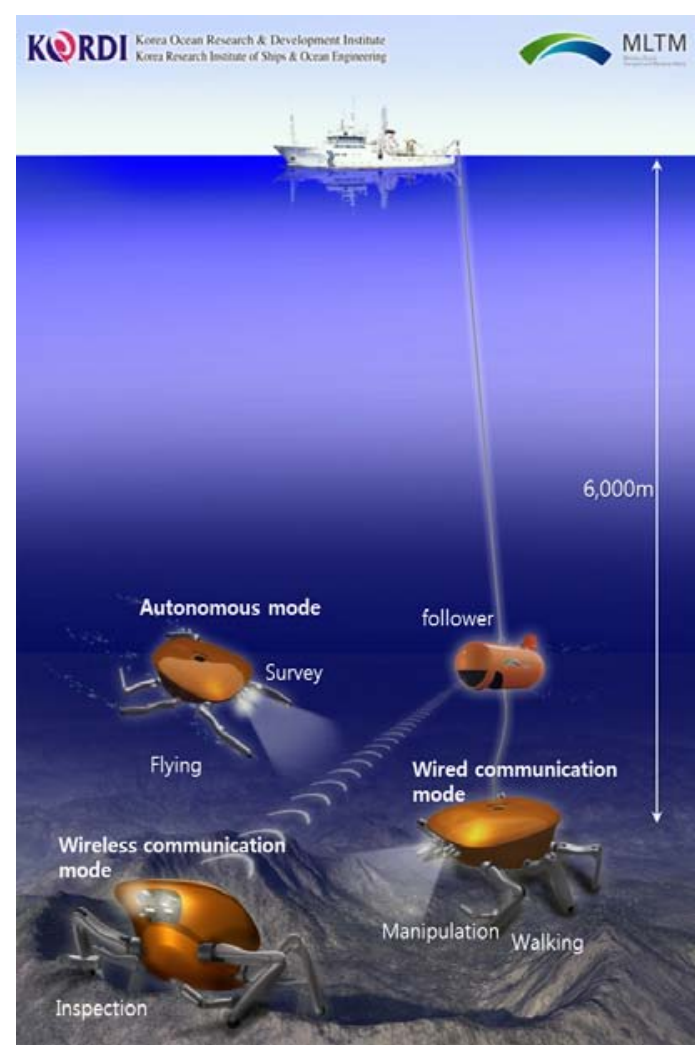

Figure 1. The concept of multi-legged seabed Crabster robot

classical N-E (Newton-Euler) mechanics in the case of the underwater manipulator. McMillan [5] developed a dynamic simulation algorithm for the underwater vehicle using a robotic manipulator based on N-E formulation. Tarn et al. [6] developed a dynamic underwater vehicle model with a manipulator using Kane's method. McLain et al. [7] conducted experiments to investigate the behavior of the added mass and drag coefficients of the motion of a single link arm and showed that the dynamic interaction between the vehicle and the arm could be significant. They also experimentally determined the added mass and drag coefficients of a two-link arm. However, neither was able to provide closed dynamic equations that were explicitly described as functions of generalized coordinates and its 1st and 2nd derivatives.

In this paper, we present the concept and main missions of the Crabster robot (Fig. 1). The main focus is on the modeling of hydrodynamic forces on the legs of the robot, which creates the main difference between the on-land and underwater robots. An approximated numerical model of the drag and lift forces acting on the articulated legs is presented. The model is described as closed-form dynamic equations based on the L-E (LagrangeEuler) formulation. Since the closed-form equations of motion have the advantages of being easy to apply and a control design purpose, the presented model guarantees the same advantages in underwater manipulators. Generalized drag torque is successfully derived as a function of generalized variables and its first derivative despite the arm having a roll joint and twist angles between the joints. To verify the proposed model, we conducted drag torque simulations using a simple Selective Compliant Articulated Robot Arm (SCARA).

\section{Underwater walking Crabster robot}

As the name implies, the Crabster is an underwater robot that mimics the moving capabilities of a crab and a lobster. In other words, Crabster can walk on sea floor like a lobster and can swim like a crab (Fig. 1). The Crabster robot will be implemented as 2 types of underwater robots: the CR200, which works in a shallow and strong tide area by crawling across the sea floor, and the CR6000, which works on deep-sea sediment without disturbing the seabed. The Crabster has 6 legs for underwater walking and working. The 2 forward legs each have 6 degrees of freedom, allowing them to function as both manipulators and legs, while the middle and rear legs have 4 degrees of freedom, which allow leg functions only. All of the joints are actively controlled by electric motors.

The CR200 endures the tidal current by controlling the posture of its streamlined body and legs based on the detected environmental information. The CR200 controls the leg joint angles so the hydrodynamic forces on the body and legs work to improve the stability margin. Generally, most of the high current environments are also high turbidity environments. The CR200 also has a real-time vision system that consists of optical and acoustic cameras for the high turbidity environments. Cable drag may be another strong disturbance to the CR200 in high current. To minimize the effect of cable drag, a depressor works as a damper between the robot and the support vessel.

In contrast, the CR6000 flies on the seabed by swimming with its legs. The adjusting mechanism 
of buoyancy enables the robot to walk and fly with minimal disturbance to the seabed sediment. To estimate the environmental status, the Crabster senses speed and direction of sea current, the contact force of each foot, and the force/torque of each leg.

Figure 1 shows the operation concept of the Crabster. As depicted in the figure, a wireless communication mode can completely isolate the CR6000 from the depressor motion. The 4 key technologies necessary to developing the proposed robot have been enumerated and approaching methods to develop the technologies have been proposed [8].

The missions of the Crabster can be summarized as follows:

1.Survey and inspection of subsea structures and shipwrecks in shallow water

- To survey subsea structures and shipwrecks in high current (up to 2 knots) environments by keeping the body close to the sea bottom.

- To inspect high turbidity environments using optical and acoustical image devices.

- To conduct underwater work that is necessary for the inspection of underwater structures and shipwrecks such as wire cutting, grinding, and drilling using manipulators.

2. Scientific research in deep-sea environments

- To gather scientific data for physical, chemical, biological, and geological research in deep-sea environments up to $6,000 \mathrm{~m}$ that minimally disturbs the seabed sediment

- To sample deep-sea creatures, soil, and water for the scientific research

One of the main technologies for both of CR200 and CR6000 is the understanding and utilization of the hydrodynamic forces on the robot. In the following section, we present the approximated modeling of the hydrodynamic forces acting on the multi-linked legs that are necessary for the robot to use the forces.

\section{Hydrodynamic forces on the legs}

One of the main strategies for developing the CR200 was modeling and utilization of the hydrostatic and hydrodynamic forces acting on the underwater body and legs. Modeling of the underwater link is presented in this section. When an articulated robot with $\mathrm{n}$ degrees of freedom moves in an underwater environment, the dynamic equation of robot can be described as

\section{$\mathbf{M}(\mathbf{q}) \ddot{\mathbf{q}}+\mathbf{C}(\mathbf{q}, \dot{\mathbf{q}})+\mathbf{D}(\mathbf{q}, \dot{\mathbf{q}}) \approx \mathbf{G}(\mathbf{q})=$}

where $\mathbf{M} \in \mathfrak{R}^{n \times n}$ is an inertia matrix including added mass, $\mathbf{C} \in \mathfrak{R}^{n}$ is Coriolis and a centrifugal term caused by a rigid body and added mass, $\mathbf{D} \in \mathfrak{R}^{n}$ is hydrodynamic drag and lift force, $\mathbf{G} \in \mathfrak{R}^{n}$ is buoyancy and gravity, and $\boldsymbol{\tau} \in \mathfrak{R}^{n}$ is a joint torque vector. The hydrodynamic drag force acting on a rigid body can be decomposed into shear drag and pressure drag. Since the shear drag will be small for the underwater robot, we handle only the pressure drag as many previous works have done. The drag term D in (1) is a function of joint angles, joint velocities, fluid velocity, and hydrodynamic coefficients determined by the link geometries. To describe the drag term explicitly, the approach starts from the link coordinate system shown in Fig. 2. Assuming that the j-th link is partitioned into thin slices with small dl thicknesses, we can approximate the drag force acting on a slice in the fluid as a vector equation represented in the $i$-th frame by

$$
d^{i} \mathbf{f}_{D j}=-\frac{1}{2} \rho C_{D j}\left(\sigma_{j}\right) d_{p j}\left\|^{i} \mathbf{v}_{j}^{n}\right\|^{i} \mathbf{v}_{j}^{n} d l
$$

where $d^{i} \mathbf{f}_{D j}$ is the drag force acting on a slice in the $j$-th link expressed in $i$-th coordinate system and

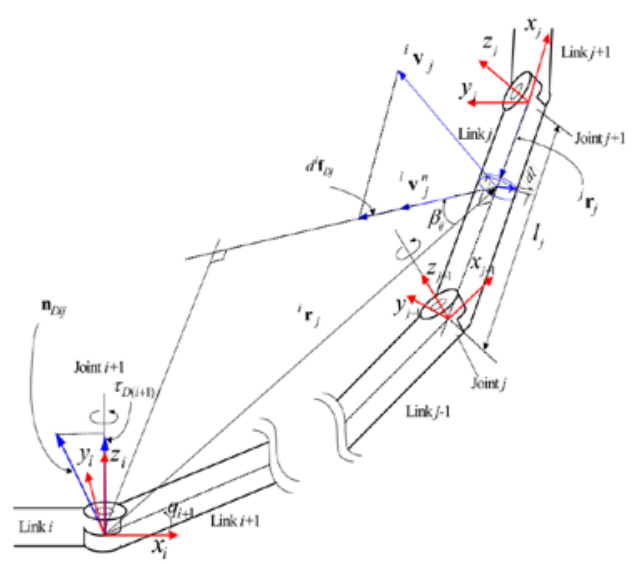

Figure 2. Vector diagram and underwater link coordinates 
$C_{D j}\left(\sigma_{j}\right)$ is the 2-dimensional drag coefficient of the $j$-th link represented as a function of the angle of the attack $\sigma_{j}$, the angle between the fluid velocity ${ }^{0} \mathbf{v}_{c}$ and the slice's velocity vector ${ }^{0} \mathbf{v}_{j}^{n}$ (Fig. 4). The $d_{p j}$ value is the projected length of the slice to the vector normal to ${ }^{i} \mathbf{v}_{j}^{n}$. The ${ }^{i} \mathbf{v}_{j}^{n}$ is the translational velocity component of the slice normal to the longitudinal axis of the $j$-th link. ${ }^{i} \mathbf{v}_{j}^{n}$ is obtained by projecting the slice velocity with respect to the fluid velocity to the $\left(y_{j}-z_{j}\right)$ plane as

$$
{ }^{i} \mathbf{v}_{j}^{n}=\mathbf{P}_{i j}\left({ }^{i} \mathbf{v}_{j}-{ }^{i} \mathbf{v}_{c}\right)
$$

where ${ }^{i} \mathbf{v}_{j}$ is the velocity of the $j$-th link expressed in the $i$-th frame and $\mathbf{P}_{i j}$ is the projection matrix to the $\left({ }^{i} y_{j}-{ }^{i} z_{j}\right)$ plane expressed in the $i$-th frame defined as

$$
\begin{aligned}
& \mathbf{P}_{i j}=\mathbf{B}\left(\mathbf{B}^{T} \mathbf{B}\right)^{-1} \mathbf{B}^{T}
\end{aligned}
$$

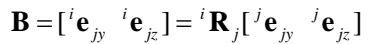

where ${ }^{i} \mathbf{R}_{j}$ is rotation matrix from the $i$-th frame to the $j$-th frame. ${ }^{i} \mathbf{e}_{j y}$ and ${ }^{i} \mathbf{e}_{j z}$ are y-axis and zaxis unit vectors, respectively, that are expressed in the $i$-th coordinate system, while ${ }^{j} \mathbf{e}_{j y}$ and ${ }^{j} \mathbf{e}_{j z}$ are expressed in the $j$-th coordinate system.

To derive the drag and lift force on the $(i+1)$-th joint as a function of joint variables and its first derivative, we first derived the velocity of a point fixed in the $j$-th link with respect to the $i$-th coordinate system. Using the notation [9] and defining the notation $(\cdot)^{\prime}$ as a transformation from a Cartesian-coordinate vector to a homogeneouscoordinate vector defined by ${ }^{i} \mathbf{r}_{j}^{\prime}=\left[{ }^{i} \mathbf{r}_{j}{ }^{T} 1\right]^{T} \in \mathfrak{R}^{4}$, we can write the velocity of the $j$ link expressed in the $i$-th coordinate system as

$$
{ }^{i} \mathbf{v}_{j}^{\prime}={ }^{i} \mathbf{A}_{0}\left[\sum_{k=1}^{j} \mathbf{U}_{j k} \dot{\mathbf{q}}_{k}\right]{ }^{j} \mathbf{r}_{j}^{\prime}=\left[\sum_{k=1}^{j}{ }^{i} \mathbf{A}_{0} \mathbf{U}_{j k} \dot{\mathbf{q}}_{k}\right]{ }^{j} \mathbf{r}_{j}^{\prime} .
$$

Then, defining a new notation as
${ }^{i} \mathbf{U}_{j k}={ }^{i} \mathbf{A}_{0} \mathbf{U}_{j k}=\left\{\begin{array}{lll}{ }^{i} \mathbf{A}_{k-1} \mathbf{Q}_{k}{ }^{k-1} \mathbf{A}_{j} & \text { for } & k \leq j \\ 0 & \text { for } & k>j\end{array}\right.$

Eq. (5) can be rewritten in simplified form as

$$
{ }^{i} \mathbf{v}_{j}^{\prime}=\left[\sum_{k=1}^{j}{ }^{i} \mathbf{U}_{j k} \dot{\mathbf{q}}_{k}\right]{ }^{j} \mathbf{r}_{j}^{\prime} .
$$

Substituting (7) into (3), we can describe the characteristic velocity ${ }^{i} \mathbf{v}_{j}^{n}$ as a function of joint variables and its first derivative by

$$
{ }^{i} \mathbf{v}_{i}^{n}=\mathbf{P}_{i j}\left(\left[\sum_{k=i}^{j}\left\{{ }^{i} \mathbf{U}_{j k} \dot{\mathbf{q}}_{k}\right\}^{j} \mathbf{r}_{j}^{\prime}\right]^{\prime \prime}-{ }^{i} \mathbf{v}_{c}\right)
$$

where $(\cdot)^{\prime \prime}$ is the inverse transformation of $(\cdot)^{\prime}$ defined by $\left({ }^{i} \mathbf{r}_{j}^{\prime}\right)={ }^{i} \mathbf{r}_{j} \in \mathfrak{R}^{3}$.

$\mathbf{P}_{i j}$ and ${ }^{i} \mathbf{U}_{j k}$ in Eq. (8) are the functions of the joint variable $\mathbf{q}$ and ${ }^{j} \mathbf{r}_{j}^{\prime}$ is the position vector from origin of $j$-th frame to the slice in link $j$. Since the $j$-th frame is fixed on the $j$-th link (Fig. 3), ${ }^{j} \mathbf{r}_{j}^{\prime}$ can be expressed as

$$
{ }^{j} \mathbf{r}_{j}^{\prime}=\left[\begin{array}{c}
-x \\
0 \\
0 \\
1
\end{array}\right]
$$

where $x$ is the distance from the $j$-th frame to a slice. As the surface integral can be replaced with a line integral using the strip theory, the drag force on the $j$-th link can be written from Eq. (2) as

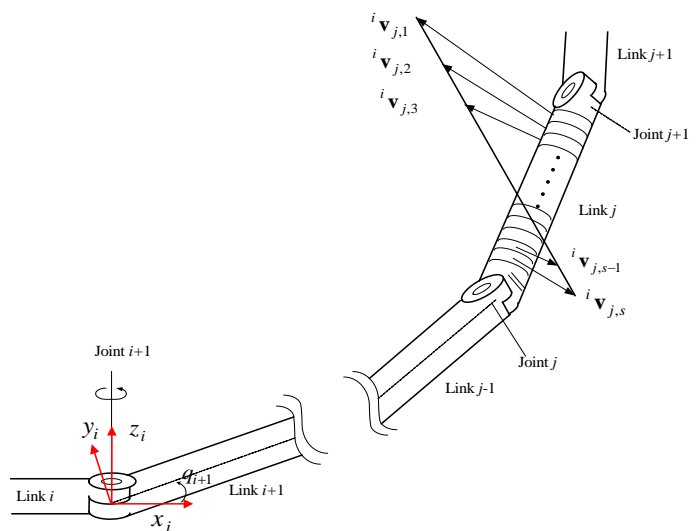

Figure 3. Strip of link and its velocity 


$$
{ }^{i} \mathbf{f}_{D j}=-\frac{1}{2} \rho \int_{0}^{l_{j}} C_{D j}\left(\sigma_{j}\right) d_{p j}\left\|^{i} \mathbf{v}_{j}^{n}\right\|^{i} \mathbf{v}_{j}^{n} d l .
$$

Assuming that the surrounding fluid is at rest for simplicity and substituting Eq. (8) into Eq. (10) and approximating the definite integral in Eq. (10) as a numerical integration, it follows that

$$
\begin{aligned}
{ }^{i} \mathbf{f}_{D j}=-\frac{1}{2} \rho C_{D j}\left(\sigma_{j}\right) d_{p j} \sum_{x=1}^{s}\left[\left\|\mathbf{P}_{i j}\left(\sum_{k=1}^{j}\left\{{ }^{i} \mathbf{U}_{j k} \dot{\mathbf{q}}_{k}\right\}^{j} \mathbf{r}_{j}^{\prime}\right)^{\prime \prime}\right\|\right. \\
\left.\mathbf{P}_{i j}\left(\sum_{k=1}^{j}\left\{\mathbf{U}_{j k} \dot{\mathbf{q}}_{k}\right\}^{j} \mathbf{r}_{j}^{\prime}\right)^{\prime \prime} \frac{l_{j}}{s}\right]
\end{aligned}
$$

$$
{ }^{j} \mathbf{r}_{j}^{\prime}=\left[\begin{array}{c}
-x l_{j} / s \\
0 \\
0 \\
1
\end{array}\right]
$$

where $s$ is the number of strips. Next, the drag torque on the $\underline{i}$-th coordinate system exerted by drag force of the $j$-th link can be written as

$$
\begin{array}{r}
\mathbf{n}_{D i j}=-\frac{1}{2} \rho C_{D j}\left(\sigma_{j}\right) d_{p j} \sum_{x=1}^{s}\left[\left\{i^{\mathbf{r}_{j} \times}\left\|\mathbf{P}_{i j}\left(\sum_{k=1}^{j}\left\{{ }^{i} \mathbf{U}_{j k} \dot{\mathbf{q}}_{k}\right\}^{j} \mathbf{r}_{j}^{\prime}\right)^{\prime \prime}\right\|\right.\right. \\
\left.\left.\mathbf{P}_{i j}\left(\sum_{k=1}^{j}\left\{{ }^{i} \mathbf{U}_{j k} \dot{\mathbf{q}}_{k}\right\}^{j} \mathbf{r}_{j}^{\prime}\right)^{\prime \prime}\right\} \frac{l_{j}}{s}\right]
\end{array}
$$

where ${ }^{i} \mathbf{r}_{j}$ is the position vector from the $i$-th frame to the $j$-th frame.

$$
{ }^{i} \mathbf{r}_{j}=\left({ }^{i} \mathbf{r}_{j}^{\prime}\right)^{\prime \prime}, \quad{ }^{i} \mathbf{r}_{j}^{\prime}={ }^{i} \mathbf{A}_{j}{ }^{j} \mathbf{r}_{j}^{\prime}={ }^{i} \mathbf{A}_{j}\left[\begin{array}{c}
-x l_{j} / s \\
0 \\
0 \\
1
\end{array}\right]
$$

Since the axis of the $i$-th joint coincides with the $Z_{i-1}$ axis of the (i-1)th coordinates, the drag torque on the $i$-th joint exerted by the $j$-th link is obtained by projecting the $\mathbf{n}_{D(i-1) j}$ to the $Z_{i-1}$ axis as

$$
\begin{aligned}
& \tau_{D i j}={ }^{i-1} \mathbf{e}_{(i-1) l_{0}^{T}}^{T} \mathbf{n}_{D(i-1) j} \\
& =-\frac{1}{2} \rho C_{D j}\left(\sigma_{j}\right) d_{p j}^{i-1} \mathbf{e}_{(i-1))}^{T} \sum_{x=1}^{s}\left[\left\{\int_{i-1} \mathbf{r}_{j} \times\left\|\left(\mathbf{P}_{(i-1) j} \sum_{k=1}^{j}\left\{i^{i-1} \mathbf{U}_{j k} \dot{\mathbf{q}}_{k}\right\}^{j} \mathbf{r}_{j}\right)^{\prime}\right\|\right.\right. \\
& \left.\left.\left.\mathbf{P}_{(i-1) j}\left(\sum_{k=1}^{j} \sum_{i=1}^{i-1} \mathbf{U}_{j k} \dot{\mathbf{q}}_{k}\right\}^{j} \mathbf{r}_{j}^{\prime}\right)^{\prime \prime}\right) \frac{l_{j}}{s}\right]
\end{aligned}
$$

where

$$
{ }^{j} \mathbf{r}_{j}^{\prime}=\left[\begin{array}{c}
-x l_{j} / s \\
0 \\
0 \\
1
\end{array}\right], \quad{ }^{i-1} \mathbf{r}_{j}^{\prime}={ }^{i-1} \mathbf{A}_{j}{ }^{j} \mathbf{r}_{j}^{\prime} \text { and }{ }^{i-1} \mathbf{r}_{j}=\left({ }^{i-1} \mathbf{r}_{j}^{\prime}\right)^{\prime \prime}
$$

Finely, the drag torque on the $i$-th joint exerted by all of the links, $\tau_{D i}$, is obtained as

$$
\tau_{D i}=\sum_{j=i}^{n} \tau_{D i j}, \quad i=1,2, \cdots, n .
$$

In the same manner, the lift torque on the $i$-th joint exerted by the $j$-th link can be obtained as

$$
\begin{aligned}
& \tau_{L i j}={ }^{i-1} \mathbf{e}_{(i-1)]_{2}^{T}}^{T} \mathbf{n}_{L(i-1) j}
\end{aligned}
$$

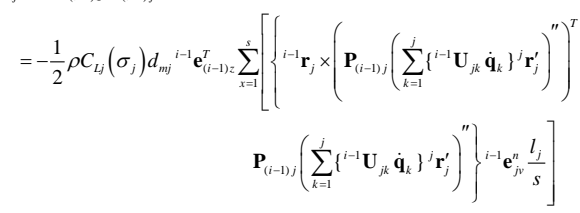

where $\tau_{L i j}$ is the lift torque on $i$-th joint exerted by the $j$-th link and $C_{L j}\left(\sigma_{j}\right)$ is the lift coefficient represented by the angle of attack $\sigma_{j}$. The $d_{m j}$ is the major axis of the cross-section of the $j$-th link and ${ }^{i-1} \mathbf{e}_{j v}^{n}$ is the unit vector normal to the plane spanned by ${ }^{i-1} \mathbf{v}_{j}^{n}$ and ${ }^{i-1} \mathbf{e}_{j x}$. Note that the ${ }^{i-1} \mathbf{e}_{j v}^{n}$ is used to specify the direction of the lift force, the sign of which is determined by the sign of the angle of attack $\sigma_{j}$. The lift torque on the $i$-th joint exerted by all the links, $\tau_{L i}$, is obtained as

$\tau_{L i}=\sum_{j=i}^{n} \tau_{L i j}, \quad i=1,2, \cdots, n$

Adding Eq. (19) to Eq. (17), the generalized damping term in Eq. (1) including drag and lift torque is obtained as

$$
\mathbf{D}(\mathbf{q}, \dot{\mathbf{q}})=-\left[\tau_{T 1}, \cdots, \tau_{T n}\right]^{T}
$$

where $\tau_{T i}=\tau_{D i}+\tau_{L i},(i=1,2, \cdots, n)$ and $n$ is the number of degrees of freedom of the leg. Equation (20) is called the generalized damping torque. 
The angle of attack $\sigma_{j}$ is the angle between the major axis of the link slice and the characteristic velocity vector ${ }^{i} \mathbf{v}_{j}^{n}$. If the offset angle between the orientation of the slice and the y-axis of the $j$-th coordinates is defined as $\sigma_{0}, \sigma_{j}$ is obtained as

$\sigma_{j}=\Gamma_{j}+\sigma_{0}$

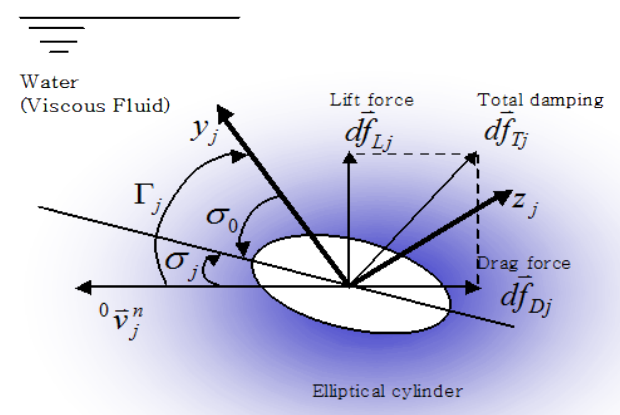

Figure 4. Definition of the angle of attack

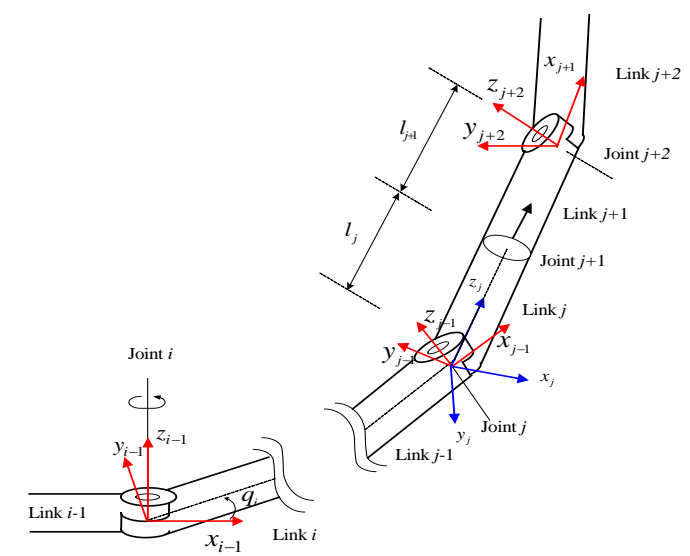

Figure 5. Multiple links with a roll joint

$$
\Gamma_{j}=\cos ^{-1}\left(\frac{\left({ }^{0} \mathbf{v}_{j}^{n}\right)^{T} \mathbf{e}_{j y}}{\left|{ }^{0} \mathbf{v}_{j}^{n} \|{ }^{0} \mathbf{e}_{j y}\right|}\right)=\cos ^{-1}\left(\left({ }^{i-1} \mathbf{e}_{j v}\right)^{T i-1} \mathbf{e}_{j y}\right)
$$

If the $(j+1)$-th joint of the manipulator is the roll joint (Fig. 5), since the $z$-axis of $j$ th coordinate system aligned to the longitudinal direction of link $j$, the projection matrix $\mathbf{P}_{i j}$ becomes

$$
\begin{aligned}
& \mathbf{P}_{i j}=\mathbf{B}\left(\mathbf{B}^{T} \mathbf{B}\right)^{-1} \mathbf{B}^{T} \\
& \mathbf{B}=\left[\begin{array}{ll}
{ }^{i} \mathbf{e}_{j x} & \left.{ }^{i} \mathbf{e}_{j y}\right]={ }^{i} \mathbf{R}_{j}\left[{ }^{j} \mathbf{e}_{j x}{ }^{j} \mathbf{e}_{j y}\right]
\end{array} .\right.
\end{aligned}
$$

and ${ }^{j} \mathbf{r}_{j}^{\prime}$ becomes

$$
{ }^{j} \mathbf{r}_{j}^{\prime}=\left[\begin{array}{c}
0 \\
0 \\
x l_{j} / s \\
1
\end{array}\right]
$$

Since $Z_{j}$-axis and ${ }^{j} \mathbf{v}_{j}^{n}$ are always normal to each other, the drag and lift force on the $j$ frame exerted by the $j$-th link is always zero, i.e., $\tau_{i i}$ is always zero.

\section{Generalized drag torque simulation}

The drag force shows the most significant difference in dynamic characteristics between the land and underwater environments when the link has relative velocity with respect to fluid. As such, the main focus of modeling was the derivation of drag force and generalized drag torque. To verify the drag model derived in this paper, numerical simulation was conducted with using a SCARA type 3-link robot (Fig. 6). The simulation results are plotted in Figs. 7 and 8. Figure 7 shows the interaction of drag torque due to the velocity of each joint when the angle of joint 3 varied from 0 to 360 degrees, while Fig. 8 shows when the angle of joint 2 varies in the same range. In Fig. 7, figure (a) shows the drag torque of each joint when the 3rd joint has unit velocity. In this case, the 3rd joint undergoes constant torque while other joints take maximum torques in absolute value at the full reach, i.e., $q_{3}=2 n \pi,(n=1,2, \cdots)$. When the 1 st joint has unit velocity, the direction of torque on joint 3 is reversed at the point at which the angle of joint 1 is approximately $100^{\circ}$ (Fig. 7 (c)). At that point, the absolute value of the torques of joints 1 and 2 go to minimum values, creating a kind of drag optimal posture. In the case of joint 2, this situation appears at the point that angle of joint 2 goes to $130^{\circ}$ (Fig. 8 (c)).

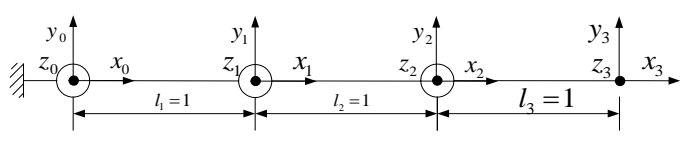

\begin{tabular}{|c|c|c|c|c|}
\cline { 2 - 5 } \multicolumn{1}{c|}{} & $\theta_{i}$ & $\alpha_{i}$ & $a_{i}$ & $d_{i}$ \\
\hline 1 & 0 & 0 & 1 & 0 \\
\hline 2 & 0 & 0 & 1 & 0 \\
\hline 3 & 0 & 0 & 1 & 0 \\
\hline
\end{tabular}

Figure 6. Coordinate system and link parameters of the 3-link Selective Compliant Articulated Robot Arm 


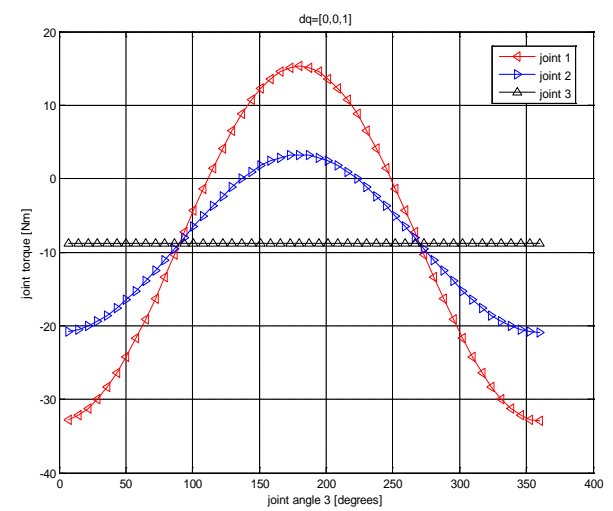

(a) Unit velocity in the 3rd joint

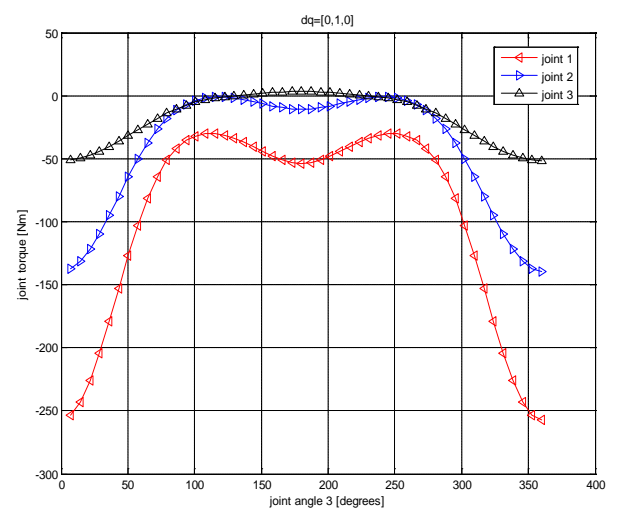

(b) Unit velocity in the 2nd joint

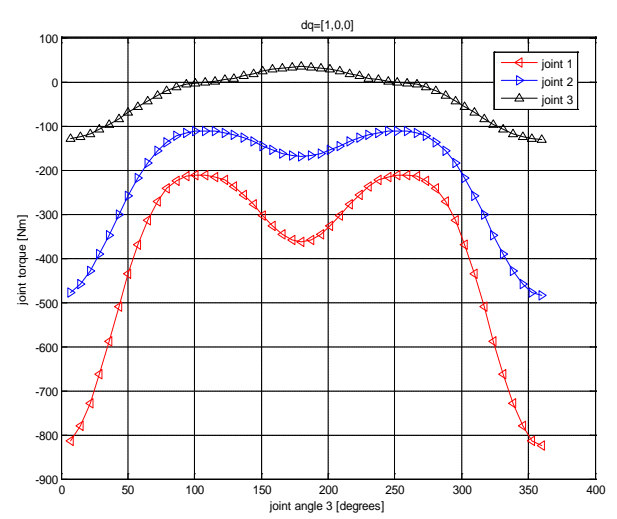

(c) Unit velocity in the 1 st joint

Fig. 7. Drag torque of each joint wrt. angle of 3rd joint.

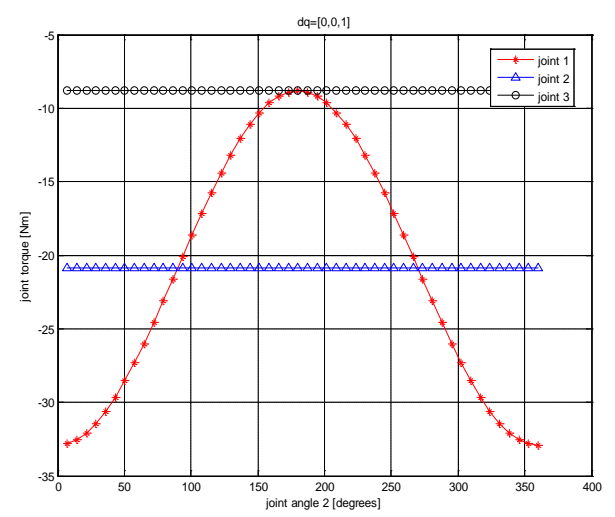

(a) Unit velocity in the 3rd joint

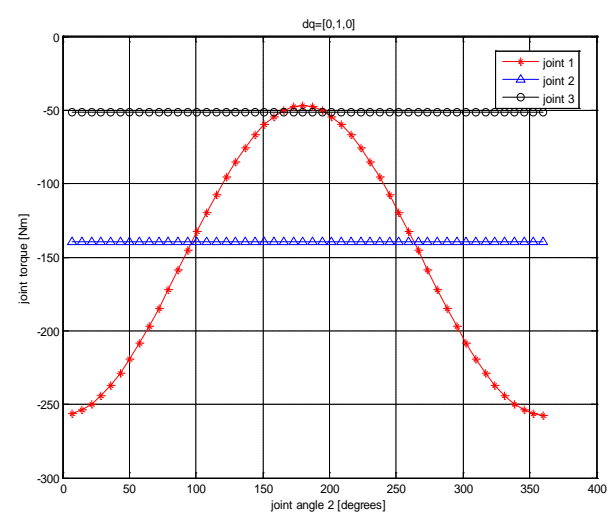

(b) Unit velocity in the 2nd joint

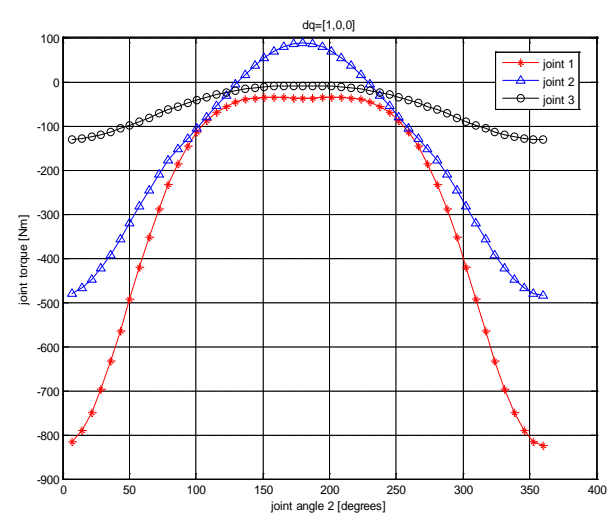

(c) Unit velocity in the 1 st joint

Fig. 8. Drag torque of each joint wrt. angle of 2nd joint 


\section{Conclusions}

In this paper, we presented an approximated model of drag and lift forces acting on articulated legs of the Crabster seabed-walking robot. Generalized drag torque is successfully derived as a function of generalized variables and its first derivative despite the arm having to roll the joint and twist angles between the joints. To verify the proposed model, we conducted drag torque simulations using the simple Selective Compliant Articulated Robot Arm. We expect that the presented model will be applied to the dragoptimized joint path planning for underwater walking or swimming.

\section{Acknowledgements}

This work was supported by the Ministry of Land, Transportation and Maritime Affairs of Korea for the development of multi-legged walking and flying subsea robots.

\section{References}

[1] Benoit L'evesque and Marc J. Richard, Dynamic analysis of a manipulator in a fluid environment, International Journal of Robotics Research, 13(3), (1994) 221-231.

[2] J. Yuh, Modeling and control of underwater robotic vehicles, IEEE Transaction on Systems, Man and Cybernetics, 20 (1990) 1475-1483.

[3] T. I. Fossen, Guidance and Control of Ocean Vehicles, Chichester, John Wiley \& Sons Ltd., 1994.
[4] K. Ioi and K. Itoh, Modeling and simulation of an underwater manipulator, Advanced Robotics, 4 (4) (1990) 303-317.

[5] S. McMillan, D. E. Orin and C. S. G. Lee, Efficient dynamic simulation of an underwater vehicle with a robotic manipulator, IEEE Transaction on Systems, Man, and Cybernetics, 25 (8) (1995) 1194-1206.

[6] T. J. Tarn, G. A. Shoults and S. P. Yang, A dynamic model of an underwater vehicle with a robotic manipulator using Kane's method, Autonomous Robots 3 (1996) 269-283.

[7] T. W. McLain and S. M. Rock, Experiments in the coordination of underwater manipulator and vehicle control, Journal of Autonomous Robots, 3 (2) (1996) 139-158.

[8] B.H. Jun, Hyungwon Shim, Jin-Yeong Park, Banghyun Kim, Pan-Mook Lee, A New Concept and Technologies of Multi-Legged Underwater Robot for High Tidal Current Environment, Proc. Of Underwater Technologies 2011, Kobe (2011).

[9] K.S. Fu, R.C. Gonzalez, and C.S.G. Lee, Robotics: Control, Sensing, Vision, and Intelligence, McGraw-Hill Book Company, (1987).

[10]B.H. Jun, P.M. Lee, J. Lee, Repetitive periodic motion planning and directional drag optimization of underwater articulated robotic arms, Int. J. of control and automation and systems, 4 (1) (2006) 42-52.

[11]B.H. Jun, P.M. Lee, H. Baek and Y.K. Lim, Approximated Modeling of Hydrodynamic Forces Acting Legs of Underwater Walking Robot, Proc. Of Oceans 2011, Santander (2011). 